\title{
HIGHLIGHTS
}

PARKINSON DISEASE

\section{Dopamine agonist alleviates depression in PD}

Clinical improvement of depressive symptoms in patients with Parkinson disease (PD) could assist in disease management. In a recent study by Paolo Barone and colleagues, a dopamine agonist, pramipexole, improved depressive symptoms after 12 weeks in European and South African patients undergoing antiparkinsonian treatment. Only patients who were not exhibiting motor fluctuations were included in the study, since such symptoms can lead to depression in individuals with PD, so treatments that stabilize motor functions could improve depressive symptoms through indirect means.

Barone et al. selected pramipexole for this study, as depression in patients with PD is thought to be linked to dopaminergic dysfunction. The research team claims that their study reports, for the first time, the efficacy of this dopamine agonist in specifically reducing depression in such individuals.
Primary and secondary outcomes were determined by the Beck Depression Inventory score and the 15-item Geriatric Depression Scale, respectively. On both these outcome measures, patients improved with pramipexole treatment compared with placebo.

\section{Clinical improvement of depressive symptoms ... could assist in disease management 77}

Path analysis indicated that the overall effect was $80 \%$ direct drug influence on depressive symptoms, attributable to stimulation of dopaminergic pathways by pramipexole, while easing of motor fluctuations accounted for $20 \%$ of the effect.

The study supports the previously held theory that an association exists between improvement in depression severity and quality of life. However, even at the beginning of the study, the participants were rated as having only mild to moderate depression (minimum baseline scores were 5 on the 15 -item Geriatric Depression Scale, and $\geq 2$ on part 1 , item 3 of the Unified Parkinson's Disease Rating Scale), so the absolute effects are small.

The international research team concludes that further investigations are warranted to determine whether improvements would be seen in patients with more-severe depressive symptoms or those with further-advanced PD, and whether the pramipexole dose can be optimized in patients who are already taking the drug to treat depressive symptoms.

\section{Carolyn McSharry}

Original article Barone, P. et al. Pramipexole for the treatment of depressive symptoms in patients with Parkinson's disease: a randomized, double-blind, placebo-controlled trial. Lancet Neurol. 9 573-580 (2010) 